\title{
Performance Analysis of CSMA, MACA and MACAW Protocols for VANETs
}

\author{
Sharanappa P. H. and Mahabaleshwar S. K.
}

\begin{abstract}
Vehicular Ad Hoc Networks (VANETs) are a special type of Mobile Ad Hoc Networks (MANETs). Recent advances in various wireless communication technologies and the emergence of computationally rich vehicles are pushing VANET research to the forefront in academia and industry. A lot of research results have been published in various areas (such as routing, broadcasting, security and others) of VANET in the last decade covering both vehicle-to-vehicle (V2V) and vehicle-to-infrastructure (V2I) scenarios. One specific area of VANET that still faces significant challenges is the design of reliable and robust media access control (MAC) protocols for V2V communications. Many algorithms of V2V MAC methods (including various VANET standards) have been proposed for VANETs over the last, few years that also focused on the benefits and limitations of the proposed MAC techniques as well as their ease of implementation in practice and future deployment. In this paper, we have made the performance analysis of Carrier sense multiple access (CSMA), Multiple Access with Collision Avoidance (MACA) and Multiple Access with Collision Avoidance for Wireless (MACAW) for VANET environment.
\end{abstract}

Index Terms-CSMA, MACA, MACAW, media access control, VANETs.

\section{INTRODUCTION}

Vehicular ad hoc networks (VANETs) have received increased industrial and research interests recently. The major drive for this development is the emergence of wireless networking technologies. VANETs are envisaged to provide a communication range of $1000 \mathrm{~m}$ with roadside units (RSUs) and other vehicles. VANET is a promising form of mobile ad hoc network, in which vehicles are equipped with radios for inter-vehicle communication. VANET enables run-time vehicle-to-vehicle (V2V) and vehicle-to infrastructure (V2I) message exchange, for better safety, fuel efficiency, comfort and entertainment and also, enables information sensing, aggregation, dissemination, and storing within sensors and vehicles in a distributed fashion.

In this paper, we focus on the performance analysis of MAC protocols like Carrier sense multiple access (CSMA), Multiple Access with Collision Avoidance (MACA) and Multiple Access with Collision Avoidance for Wireless (MACAW) for VANET environment. So, we propose a discrete model for performance evaluation of MAC-broadcasting protocols, based on basic ideal of literature for VANETs highway scenario. Through the

Manuscript received July 15, 2015; revised October 31, 2013.

The authors are with the Electronics and Communication Department, Basaveshwar Engineering College, Bagalkot-587102, Karnataka, India (e-mail: phsharanu@gmail.com,mahabalesh_sk@yahoo.co.in). analysis, we can study the relationship between groups of parameters, namely contention windows, hidden/exposed vehicle nodes, communication reliability, system throughput and broadcasting delay.

The remainder of this paper is organized as follows. In Section II we present some related works and discuss several key technical challenges for providing reliable MAC protocol for VANETs. We discuss the MAC protocols for VANETs and proposed scheme in Section III. The simulation scheme is presented in Section IV. We draw conclusion in Section V.

\section{LITERATURE SURVEY}

The research work in [1], the network is divided into cells characterized by one CDMA code while inside each cell an SRMA protocol is used thereby allowing realization of a collision free transmission. Inside a cell the messages are sent according to SOTDMA protocol where nodes send their messages in their already reserved slots and reserve a free slot in a next frame. When a node is about to cross a cell border, it must observe the "slot frame" of the target cell and reserve one of the first six free slots according to its current's cell priority.

The work in [2] is a modified analytical model based on Markov chain for IEEE 802.11 broadcast to derive closed form expressions of the saturation throughput, the saturation delay, and the packet reception ratio. Several important performance indices are derived from the proposed analytical model taking IEEE 802.11 standard MAC broadcasting protocol and saturation traffic into account. The scheme given in [3] is based on a hybrid channel access mechanism exploiting both the advantage of TDMA and CSMA/CA. Adaptive broadcasting provides collision-free and delay bounded transmissions for safety messages, and it enhances the adaptability of the MAC protocol to different traffic density conditions.

The work in [4], qualitatively compares some MANETs MAC protocols that can be used in VANETs, since vehicles move very fast, the topology of the network changes rapidly and often. Thereby routing in inter-vehicular networks is a difficult task. Low latency and high reliability must be also taken into account because of active safety applications.

The work in [5], the source node must first complete the collision avoidance phase. Then source node broadcasts RTS to its neighbors and sets the WAIT-FOR-CTS timer. Upon receiving the RTS, each neighbor transmits CTS if it is not in YIELD state and sets the WAITFOR-DATA timer. If the source node receives CTS before the WAIT-FOR-CTS timer expires, the node transmits DATA. Otherwise, the source 
node backs off and retransmits at a later time. Any neighbor of the source node that did not receive DATA within the expected WAIT-FOR-DATA time period transmits NAK to the source. If the source node does not receive NAK after the WAIT-FOR-NAK timer expires, the source node assumes that all the neighbors have successfully received DATA. Otherwise, the source node will back off and retransmit DATA at a later time. But this protocol doesn't guarantee the delivery of broadcast packets.

A new MAC protocol called RMAC that supports reliable multicast for wireless ad hoc networks using the busy tone mechanism is proposed in [6]. It focuses on using ARQ to implement the MAC layer reliable multicast for wireless ad hoc networks where the number of one-hop multicast receivers is not large. RMAC achieves high reliability with very limited overhead.

In [7], it illustrates the design of a cross-layered MAC and clustering solution for supporting the fast propagation of broadcast messages in VANET. A distributed dynamic clustering algorithm is proposed to create a dynamic virtual backbone in the vehicular network. The vehicle-members of the backbone are responsible for implementing efficient messages propagation.

Markov chains and stochastic process is proposed to obtain the proper minimum contention window [8]. The work in [9] presents a survey of V2V MAC methods. It focuses on the benefits and limitations of the proposed MAC techniques as well as their ease of implementation in practice and future deployment. In addition some of the challenges that still need to be addressed to enable the implementation of highly efficient and high performance MAC protocols for $\mathrm{V} 2 \mathrm{~V}$ communications are discussed.

The QoS supported multi channel MAC scheme can adaptively tune the contention window for different services at each node, and dynamically adjust the intervals of the Control Channel and the Service Channels working in multi-rate. The work presented in [10] uses a Dynamic TDMA mechanism that can be easily adapted to the UMTS Terrestrial Radio Access Time Division Duplex (UTRA-TDD). It is able to provide prompt access, reliable channels and support for QoS and offers high performance in terms of access delay and radio resources reuse when a single hop broadcast service is considered.

The scheme [11] proposes a comparison between different MAC protocols for wireless ad hoc networks. Specifically they consider a well-known protocol adopted at the MAC layer. It applies a random function to select a broadcast slot that represents a kind of permission for the node to reserve data slots and to send updated schedules. A topology-transparent broadcast protocol that uses positive orthogonal codes as its transmission patterns and provides a detailed mathematical analysis for obtaining the probability of success and the average delay [12]. The method [13] dynamically adjusts the intervals of Control Channel $(\mathrm{CCH})$ and Service Channels (SCHs). Markov modeling is conducted to optimize the intervals based on the traffic condition and helps in improving the saturation throughput while maintaining the prioritized transmission of critical safety information on the $\mathrm{CCH}$.

In [14], a non-interfering busy-tone signal in a short period of time is used in order to notify all hidden terminals without blocking a large number of nodes for a long time. This protocol minimizes the negative impact of both the hidden-terminal and the exposed-terminal problems with the assistance of an out-of-band busy tone signal. In [15], a new Enhanced Busy-tone Multiple Access (EBTMA) medium access control (MAC) protocol is proposed. It minimizes the negative impact of both the hidden-terminal and the exposed-terminal problems with the assistance of an out-of-band busy tone signal. The work discussed in [16] addresses the issue of emergency message dissemination in VANETs. Main focus lies in reducing broadcast delay, which is a crucial factor in time-critical safety applications. The scheme aims at lowering the contention delay incurred in one hop in an effort to allow significant reduction in the total broadcast delay. Four reliability metrics for one-hop broadcast communication in VANETs, including the packet reception rate (PRR), the packet delivery ratio (PDR), the node successful packet delivery probability (PDP), and the effective range (ER), are introduced and evaluated analytically across vehicle density, data rate and distance to sender carrier sensing range and by simulations [17].

An interleaving approach to transmit beacon frames to determine backbone vehicles to guarantee the network connectivity is presented in [18]. It dynamically selects proper vehicles to form a connected virtual backbone for provision of efficient broadcasting in VANETs. In [19], the Distributed Adaptive MAC protocol called DA-MAC protocol exploits an interleaving approach to transmit beacon frames to determine backbone vehicles to guarantee the network connectivity. The protocol in [20] supports efficient one-hop and multi-hop broadcast services on the control channel by using implicit acknowledgments and eliminating the hidden terminal problem. The protocol reduces transmission collisions due to node mobility on the control channel by assigning disjoint sets of time slots to vehicles moving in opposite directions and to road side units.

The scheme given in [21], uses beacon message to find the next relaying node. Each node's location information embedded in its beacon message, each node will have knowledge of its neighbors. This neighbor information is very useful for retrieving many useful data such as vehicle density, link reliability, transmission radius etc. In our propose work information broadcasted in the minimum hop to maximum distance. Each node calculates the no of nodes covered by their transmission range except those nodes, which is covered by the broadcaster.

\section{MAC PROTOCOLS FOR VANETS}

The most important requirements for a MAC protocol for VANETs are low latency and high reliability. The bandwidth is not so important because, for active safety measures, only small messages must be sent. But these messages must be sent quickly and with very low failure rates. Consequently, the MAC layer must be accomplished in a distributed way. Moreover, ad-hoc networks allow stations to move which introduces more complexity, as it causes permanent network changes. This may significantly impact on the MAC layer's performance. 


\section{A. Network Environment}

Proposed scheme considers a VANET scenario as shown in Fig. 1. We assume some communicating devices called vehicles, spread throughout the network (Ad hoc) randomly. Here vehicles are termed as nodes. Each node is limited by its transmission range, mobility, buffer memory, cache memory $\&$ bandwidth utilized. Source nodes always use one or more intermediate nodes to communicate with the intended node. The nodes, which lie within the transmission range of a node, are said to be neighboring nodes for that particular node.

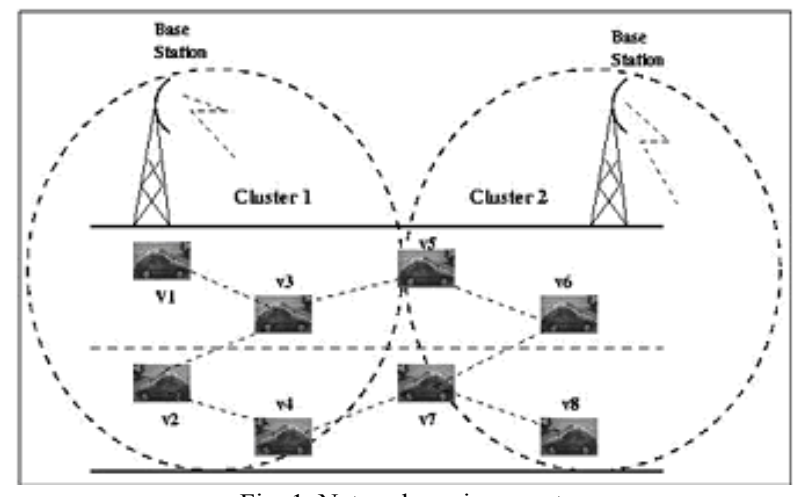

Fig. 1. Network environment.

The node, which is in need to communicate with some other node, is termed as source node, with which the source node wants to communicate is termed as destination node. Each cluster is associated with the nodes, which can form the direct connection among themselves. There will be a link between clusters by the neighboring nodes. For any source to destination there may be many paths to reach. Any node in any cluster can reach any node in the other cluster through the intermediate nodes.

\section{B. Performance Analysis}

Here in this, three MAC protocols for VANETs CSMA, MACA and MACAW are evaluated for VANETs. The performance of all the protocols is studied for different performance metrics against various parameters like number of nodes, varying mobility, etc.

CSMA: The term "Carrier Sense" signifies the capability of the terminal to listen to the channel and find out whether it is busy or not. At first sight it seems that with CSMA one can succeed in avoiding collisions altogether. Indeed, if all terminals transmit their packets only when the channel is not busy and pick a random retransmission time if they find the channel busy, then it seems that a collision will occur only when two or more terminals begin transmission simultaneously, an event that is quite unlikely. However, the situation is not as rosy as it seems, due to the finite time it takes for a signal to propagate from one terminal to another. The modified CSMA system, whose principles of operation were described above, comes by the name CSMA/CA, where CA stands for Collision Avoidance. The acronym signifies that collisions are sought to be avoided and not that they are avoided altogether. Due to the retransmission policy of the CSMA system, collisions that may occur are not detrimental: in case of collision, the ACK message or RTS CTS messages will not be received and the transmitting terminal will defer its transmission for a later time. However, if the propagation delays are relatively large and the system is heavily loaded, collisions may degrade the performance of the system.

MACA: MACA does not make use of carrier-sensing for channel access. It uses two additional signaling packets: the Request-To-Send (RTS) packet and the Clear-To-Send (CTS) packet. When a node wants to transmit data packet, it first transmits an RTS packet. The receiver node, on receiving the RTS packet, if it is ready to receive the data packet, transmits a CTS packet. Once the sender receives the CTS packet without any error, it starts transmitting the data packet. If a packet transmitted by a node is lost, the node uses the binary exponential back-off (BEB) algorithm to back-off for a random interval of time before retrying. In the BEB mechanism each time a collision is detected, the node doubles its maximum back-off window. Neighbor nodes near the sender that hear the RTS packet do not transmit for a long enough period of time so that the sender could receive the CTS packet. Both the RTS and the CTS packets carry the expected duration of the data packet transmission. A node the receiver, upon hearing the CTS packet, defers its transmission till the receiver receives the data packet. Thus, MACA overcomes the hidden terminal problem. Similarly, a node receiving an RTS defers only for a short period of time till the sender could receive the CTS. If the node hears NO CTS during its waiting period, it is free to transmit packets once the waiting interval is over. Thus a node that hears only the RTS packet is free to transmit simultaneously when the sender of the RTS is transmitting data packets. Hence the exposed terminal problem is also overcome in MACA.

MACAW: The MACAW protocol has been designed based on four main observations. The first is that the relevant congestion occurs at the receiver node and not at the sender. This realization makes CSMA protocols unsuitable for ad hoc networks, and therefore the RTS-CTS-DATA exchange mechanism of MACA becomes necessary. MACAW further improves upon this scheme using the RTS-CTS-DS-DATA-ACK exchange mechanism. The second observation is that congestion is dependant on the location of the receiver. Therefore, instead of characterizing back-off by a single back-off parameter, separate back-off parameters have been introduced for each flow. The third is that learning about congestion at various nodes must be a collective enterprise. Therefore, the notion of copying back-off values from packets has been introduced in MACA. And the final observation is that in order that nodes contend effectively for the channel, the synchronization information needs to be propagated to the concerned nodes at appropriate times. This is done in MACAW through DS and RRTS packets. Because of the changes described above, the performance of MACAW is significantly improved when compared to the MACA protocol.

\section{Simulation}

The performance analysis is done by simulating various network scenarios using $\mathrm{C}++$ language. For the Simulation of VANET, an area of $A \times B$ square meters is taken into consideration. Maximum number of nodes considered for the simulation is max_nodes. The maximum buffer size is taken 
to be max_buf. Maximum bandwidth is taken to be max_bw. Maximum cache memory of max_chm is considered. In order to simulate, the vehicles are assumed to be moving with a mobility of mobi Kms/hour. Data packets taken for transmission are assumed to be inf.

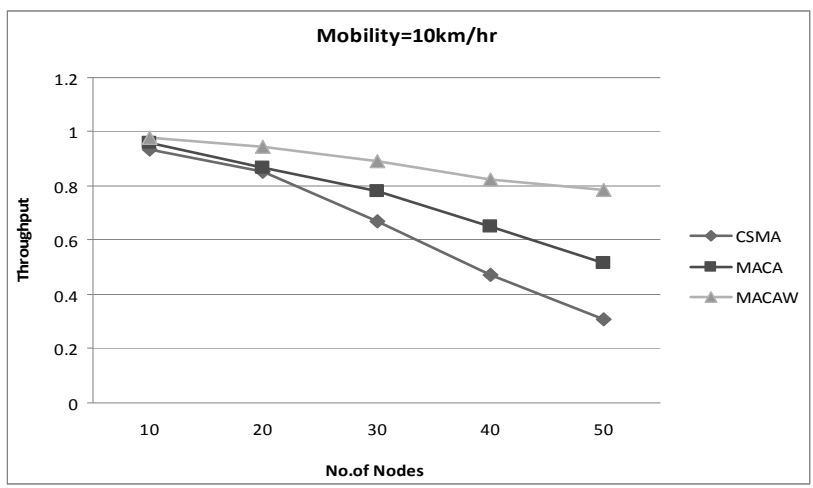

Fig. 2. Throughput vs. no. of nodes.

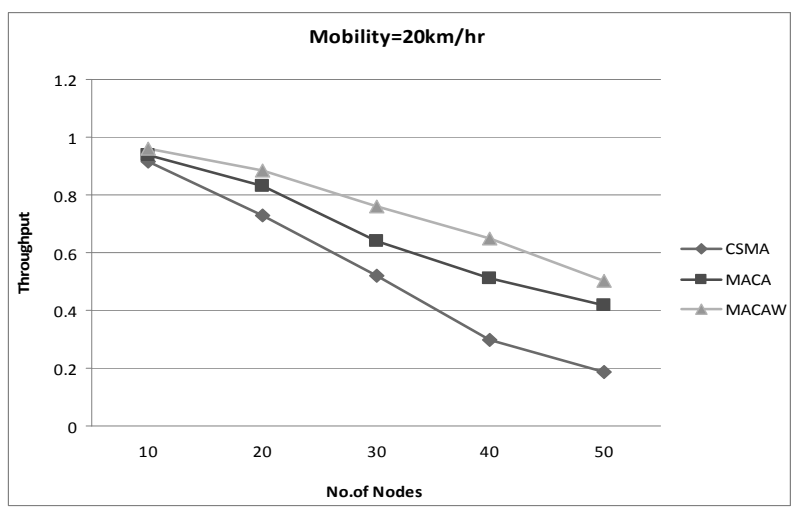

Fig. 3. Throughput vs. no. of nodes.

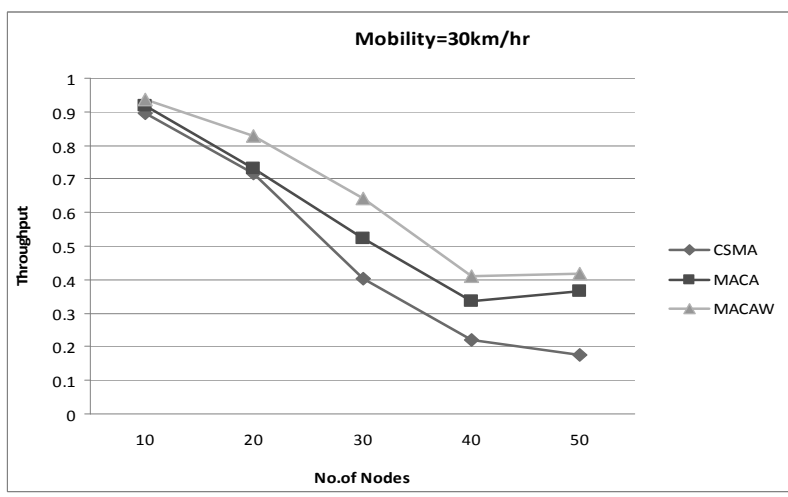

Fig. 4. Throughput vs. no. of nodes.

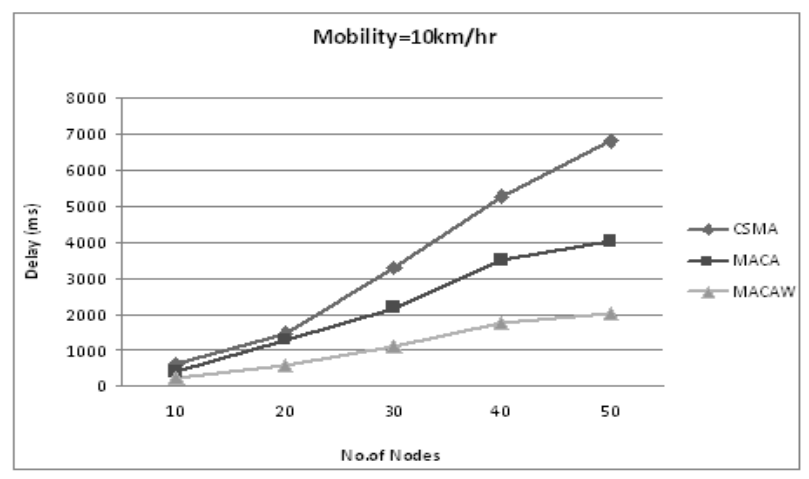

Fig. 5. Delay vs. no. of nodes

\section{A. Simulation Inputs}

To illustrate the results of the proposed VANET simulation, the simulation inputs are as follows: Dimensions for the area considered are $A=10 \mathrm{~m}$ and $\mathrm{B}=100 \mathrm{~m}$, max_nodes $=100, \quad$ max_buf $=10 \mathrm{MB}, \quad \max \_b w=100 \mathrm{MHz}$, max_chm $=500 \mathrm{~KB}$, mobi=10 Kms/Hr, inf $=10000$.

\section{B. Performance Metrics}

The performances of all the above-discussed protocols are analyzed on a common platform for various metrics. The performance parameters considered are as follows:

- Delay: It is defined as the time taken for the data packet to reach from the transmitter to the receiver. It is expressed in seconds.

- Throughput: It is defined as the ratio of number of packets received (delivered) at the receiver to the total number of packets transmitted.

- Collisions: It is defined as the ratio of number packets getting collided to the total number of packets transmitted. It is expressed in terms of percentage.

- Control Overhead: It is defined as the ratio of the bandwidth required for carrying out the control procedure before transmitting the data to the total available bandwidth. It is expressed as percentage.

\section{Result Analysis}

Fig. 2, 3 and 4 shows the throughput (packet delivery ratio) against number of nodes for varying mobility conditions i.e., $10 \mathrm{~km} / \mathrm{hr}$ to $30-\mathrm{km} / \mathrm{hr}$ mobility respectively. It is clear from the graph that throughput for all protocols decreases with increase in number of nodes and also throughput is maximum for MACAW when compared MACA which is more than CSMA.

The delay is maximum for CSMA, when compared to MACA, which is more than MACAW. This is shown in Fig. 5,6 and 7 for varying mobility conditions i.e., $10 \mathrm{~km} / \mathrm{hr}$ to $30-\mathrm{km} / \mathrm{hr}$ mobility respectively.

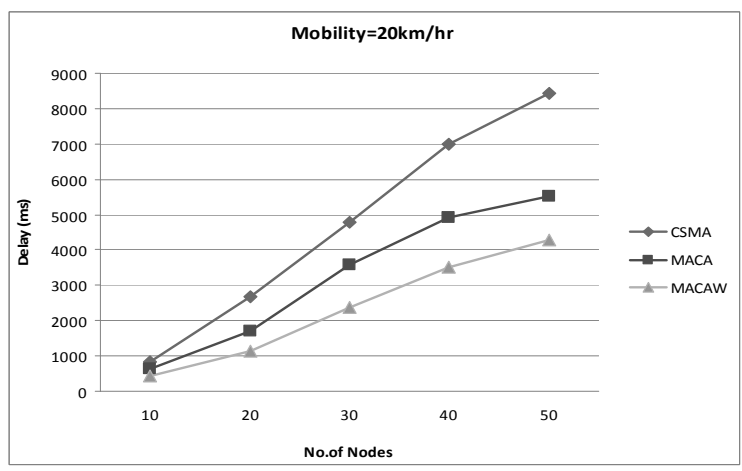

Fig. 6. Delay vs. no. of nodes

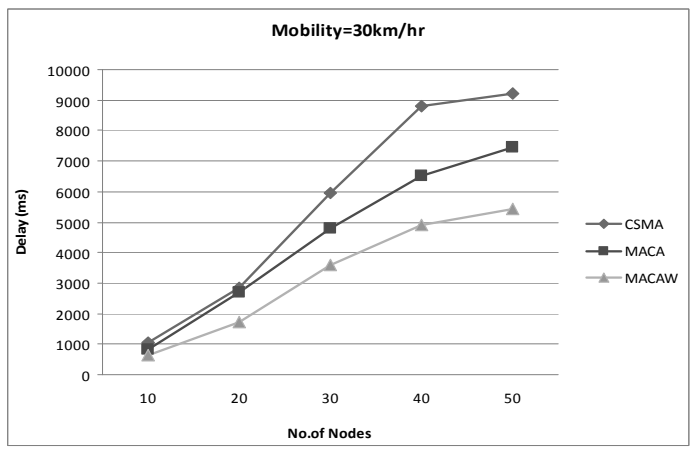

Fig. 7. Delay vs. no. of nodes. 
Fig. 8, 9 and 10 shows the number of packet collisions against number of nodes for varying mobility conditions i.e., $10 \mathrm{~km} / \mathrm{hr}$ to $30-\mathrm{km} / \mathrm{hr}$ mobility respectively. As the number of nodes increases then collisions also increase. Collisions are least in MACAW when compared to MACA, which is lesser than CSMA.

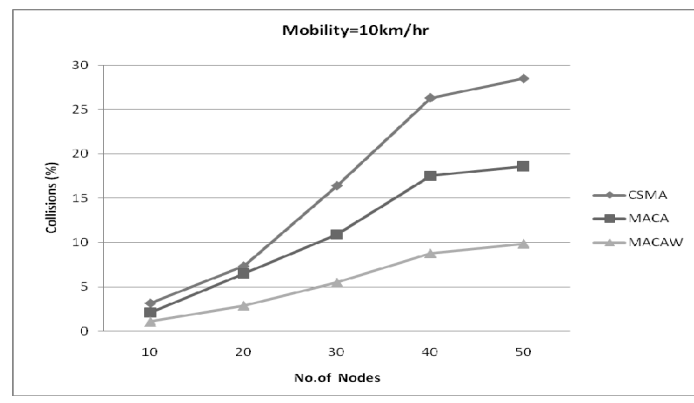

Fig. 8. Collisions vs. no. of nodes.

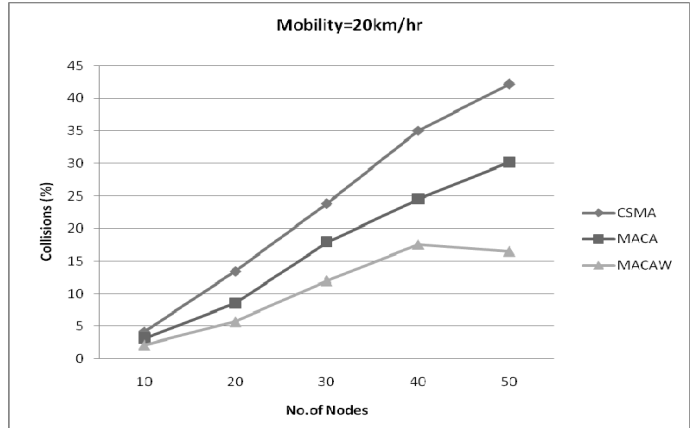

Fig. 9. Collisions vs. no. of nodes.

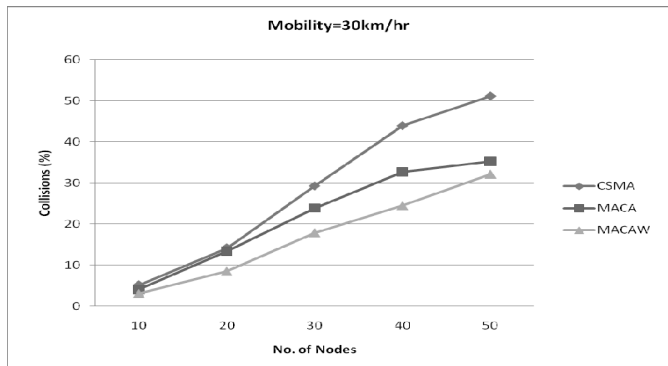

Fig. 10. Collisions vs. no. of nodes.

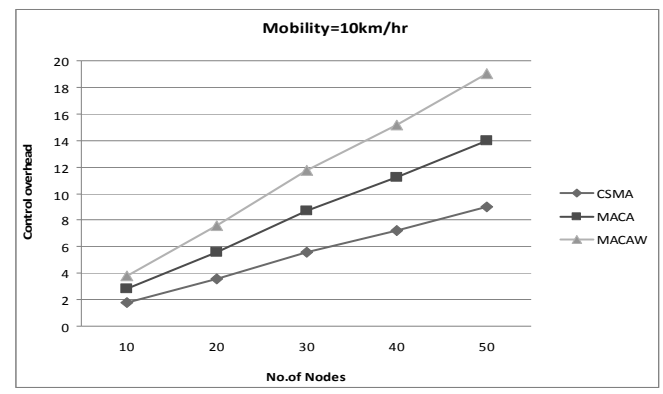

Fig. 11. Control overhead vs. no. of nodes.

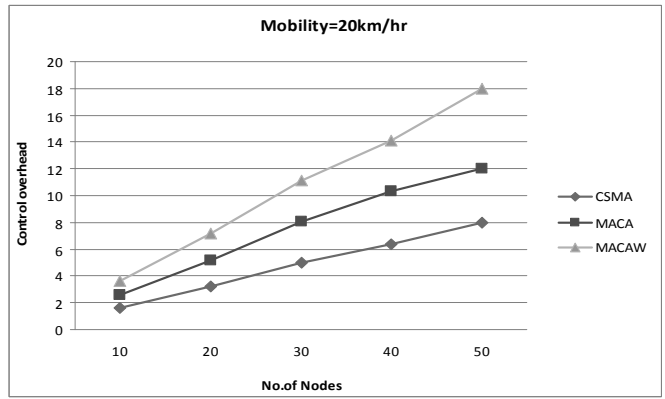

Fig. 12. Control overhead vs. no. of nodes
Control overhead is more for MACA than CSMA since it use RTS and CTS. This is depicted in Fig. 11, 12 and 13 for varying mobility conditions i.e., $10 \mathrm{~km} / \mathrm{hr}$ to $30-\mathrm{km} / \mathrm{hr}$ mobility respectively.

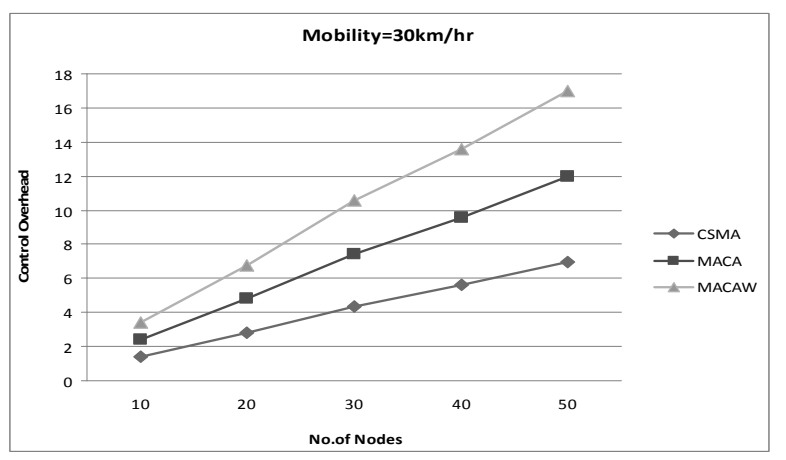

Fig. 13. Control overhead vs. no. of nodes

\section{CONCLUSION}

In this paper, we have presented a performance analysis of CSMA, MACA and MACAW. Compared to CSMA and MACA, it is clear that the MACAW is the more suitable MAC protocol for VANET conditions. But control overhead is slightly higher as compared to the CSMA and MACA. As for as CSMA is concerned; it may be having least control overhead, but also its throughput. So it depends on the application, the time constraint and the criticalness of the scenario being under study as to which protocol is to be considered.

\section{ACKNOWLEDGMENT}

We are very much thankful to TEQIP phase II of World Bank of Basaveshwar Engineering College (Autonomous), Bagalkot, Karnataka, India for sponsoring the Registartion, TA and DA expenses for attending 5th International Conference on Information and Multimedia Technology (ICIMT 2013), 6-7 December 2013, Sydney, Australia.

\section{REFERENCES}

[1] C. R. Garca, A. Lehner, and T. Strang, "A reliable MAC protocol for broadcast VANETs," in Proc. 7th International Conference on Inteligent Transportation Systems Telecommunication (ITST 2007), 6-8 June 2007, Sophia Antipolis, France.

[2] H. F. Liu, C. L. Ma, J. M. Tan, T. Wang, and Z. J. Yuan, "Modeling and analysis of mac-Broadcasting in vanets," in Proc. 5th International Conference on Wireless Communications, Networking and Mobile Computing (wicom '09), 24-26 Sept. 2009 ieee, pp. 1-4, Beijing.

[3] N. Lu, Y. S. Ji, F. Q. Liu, and X. H. Wang, "A dedicated multi-channel MAC protocol design for VANET with adaptive broadcasting," in Proc. Wireless Communications and Networking Conference (WCNC), 18-21 April, 2010 IEEE, pp. 1-6.

[4] A. Dorin, "MAC protocols for VANETs: A survey and qualitative analysis," WS 2009-2010.

[5] K. Tang and M. Gerla, "Random access MAC for efficient broadcast support in Ad Hoc networks," in Proc. Wireless Communications and Networking Confernce, (WCNC. 2000), 2000 IEEE, vol. 1, pp. 454 $-459$.

[6] W. S. Si and C. Z. Li, "RMAC: A reliable multicast MAC protocol for wireless Ad Hoc networks," in Proc. International Conference on Parallel Processing (ICPP'04), 2004, Montreal, Quebec, Canada, pp. 494-501.

[7] L. Bononi and M. D. Felice. "A cross layered MAC and Clustering scheme for efficient broadcast in VANETs," in Proc. IEEE Internatonal Conference on Mobile Adhoc and Sensor Systems(MASS 2007), 8-11 Oct. 2007, pp. 1-8. 
[8] Q. Wang, S. P. Leng, Y. Zhang, and H. R. Fu, "A QoS supported multi-channel MAC for vehicular Ad Hoc networks," in Proc. Vehicular Technology Conference (VTC Spring), 15-18 May 2011, IEEE 73rd, pp. 1-5, Budapest.

[9] M. J. Booysen, S. Zeadally, and G. J. V. Rooyen, "Survey of media access control protocols for vehicular ad hoc networks," IET Communications, pp. 1619-1631, vol. 5, no. 11, 2011.

[10] F. Borgonovo, L. Campelli, M. Cesana, and L. Coletti, "MAC for ad-hoc inter-vehicle network: Services and performance," in Proc. $58^{\text {th }}$ Vehicular Technology Conference (VTC 2003) Fall, 6-9 Oct. 2003 IEEE, vol. 5, pp. $2789-2793$.

[11] C. Y. Chiu, E. H. K. Wu, and G. H. Chen, "A reliable and efficient MAC layer broadcast protocol for mobile Ad Hoc networks," IEEE Transactions on Vehicular Technology, pp. 2296-2305, vol. 56, no. 4, July 2007.

[12] V. Loscri, "MAC schemes for ad-hoc wireless networks," in Proc. $66^{\text {th }}$ Vehicular Technology Conference, 2007. VTC-2007 Fall. IEEE, pp. 36-40.

[13] F. Farnoud and S. Valaee, "Reliable broadcast of safety messages in vehicular Ad Hoc networks," INFOCOM 2009, 19-25 April 2009, IEEE, pp. 226-234, Rio De Janeiro.

[14] Q. Wang, S. P. Leng, H. R. Fu, Y. Zhang, and H. Weerasinghe, "An enhanced multi-channel MAC for the IEEE 1609.4 based Vehicular Ad Hoc Networks," in Proc. Infocom IEEE Conference on Computer Communications Workshops , 2010, 15-19 March 2010, pp. 1-2, San Diego, CA.

[15] A. A. Abdullah, L. Cai, and F. Gebali, "Enhanced busy-tone-assisted MAC Protocol for wireless Ad Hoc networks," in Proc. $72^{\text {nd }}$ Vehicular Technology Conference Fall (VTC 2010-Fall), 6-9 Sept. 2010, IEEE, pp. 1-5, Ottawa, ON.

[16] T. Batsuuri, R. J. Bril, and J. Lukkien, "Performance and fairness in VANETs," in Proc. International Conference on Consumer Electronics (ICCE-2011), 9-12 JAN. 2011, IEEE, pp.637-638, Las Vegas, NV.

[17] J. Sahoo, E. H. K. Wu, P. K. Sahu, and M. Gerla, "Binary-Partition-Assisted MAC-Layer broadcast for emergency message dissemination in VANETs," IEEE Transactions on Intelligent Transportation Systems, pp. 757-770, vol. 12, no. 3, Sept. 2011.
[18] X. M. Ma, J. S. Zhang, and T. Wu, "Reliability analysis of one-hop safety-critical broadcast services in VANETs," IEEE Transactions on Vehicular Technology, vol. 60, no. 8, pp. 3933-3946, Oct. 2011.

[19] S. S. Wang, H. C. Chen, and J. K. Chang, "A distributed adaptive MAC protocol for efficient broadcasting in vehicular Ad Hoc networks," in Proc. Wireless Communications and Networking Conference (WCNC), 1-4 April 2012 IEEE, pp. 1555 - 1560, Shanghai.

[20] M. Khabazian, S. Aïssa, and M. M. Ali, "Performance modeling of safety messages broadcast in vehicular Ad Hoc networks," IEEE Transactions on Intelligent Transportation Systems, pp. 380 - 38, vol. 14, no. 1, March 2013.

[21] H. A. Omar, W. H. Zhuang, and L. Li, "VeMAC: A TDMA-Based MAC protocol for reliable broadcast in VANETs," IEEE Transactions on Mobile Computing, 2012.

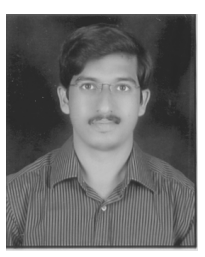

Sharanappa P. H. completed his B. E in electronics and communication engineering from visvesvaraya technological University Belgaum, India and pursuing his M.Tech in digital electronics and communication from visvesvaraya technological University Belgaum, India. Presently, he is working as faculty in Department of Electronics and Communication Engineering, Basaveshwar Engineering College, Bagalkot, Karnataka, India. He has published 2 international conference papers. His areas of interest are wireless networks. He is a member of IETE India.

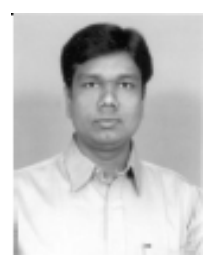

Mahabaleshwar S. K. completed his B. E in electronics and communication engineering from Karnataka University Dharwad, India, M. Tech and Ph. D. from visvesvaraya technological University Belgaum, India. Presently, he is working as Associate Professor in Department of Electronics and Communication Engineering, Basaveshwar Engineering College, Bagalkot, Karnataka, India. He has published 20 national and international conference papers and 8 national and international journals. Recently, he has coauthored a book on Wireless and Mobile Networks: Concepts and Protocols, published by Wiley-India. His areas of interest are wireless networks, especially vehicular ad hoc networks, and sensor networks. He is a member of IEEE USA, IETE India. 